\title{
Low Complexity Self-coherent Transceivers for Metro, Access and Inter-datacenter Applications
}

\author{
R. I. Killey ${ }^{(1, *)}$, M.S. Erkılınç(2), W. Yi( ${ }^{(1)}$, and P. Bayvel ${ }^{(1)}$ \\ (1) Optical Networks Group, Department of Electronic and Electrical Engineering, UCL (University College London), Torrington Place, \\ London WC1E 7JE, UK \\ (2) Submarine and Core Systems Group, Photonic Networks and Systems Dept., Fraunhofer HHI, Berlin, Germany \\ *r.killey@ucl.ac.uk
}

\begin{abstract}
Low-complexity transceivers offering high spectral efficiency and dispersion tolerance will be required for short-haul links operating at $100 \mathrm{~Gb} / \mathrm{s}$ per wavelength and beyond. Recent developments in self-coherent transceivers are described and future research directions suggested. OCIS codes: (060.4510) Optical communications; (060.4080) Modulation
\end{abstract}

\section{Introduction}

Ongoing research focuses on developing optical transceiver technologies suitable for the short-reach fiber links (typically $\leq 80 \mathrm{~km}$ ) used in metro and access networks, and interconnecting data center clusters. The simplest approach, intensity modulation with direct detection (IM/DD), offers limited information spectral density and suffers from penalties due to the chromatic dispersion of the fiber. Increased dispersion tolerance and ISD can be achieved using dual-polarization quadrature amplitude modulation (DP-QAM) with intradyne detection, however, at increased cost. An intermediate solution is the self-coherent approach, which offers some of the advantages of the coherent approach, but with reduced complexity and potentially lower cost.

In this paper, this technology is reviewed, with some recent advances described, focusing on systems with low hardware complexity (single-polarization systems, with dual-drive Mach-Zehnder modulator-based transmitters and single photodetector receivers).

\section{SSB modulation}

In self-coherent systems, an optical pilot tone is transmitted through the fiber together with the signal. Typically, the pilot tone is separated in frequency from the upper or lower edge of the signal spectrum, and acts as the phase reference at the receiver, allowing the amplitude and phase modulation of the signal to be detected through the beating between signal and pilot tone in the photodiode (i.e. heterodyne detection). Such signaling is also referred to as single-sideband (SSB) modulation, in which case the pilot tone is referred to as the optical carrier. This approach avoids the frequency-dependent fading that occurs with double sideband signals, caused by the chromatic dispersion of the transmission fiber. Electronic dispersion compensation (EDC) can be performed at the receiver, or, in the case of orthogonal frequency division multiplexing (OFDM), a cyclic prefix can be used to achieve dispersion tolerance. Key challenges are the generation of the signal and pilot tone, and the removal of the signal-signal beating terms which are generated during the photodetection.

Fig. 1 shows three different transmitter configurations that have been used to generate SSB signals. In the first (Fig. 1(a)), the signal is generated using a Cartesian IQ modulator, and the optical carrier is generated separately using a second IQ modulator, driven by sinusoidal signals with a $90^{\circ}$ phase difference, to shift the laser output to the required frequency, at one side of the signal spectrum [1]. The signal and optical carrier are combined using a coupler before transmission. The second approach, shown in Fig. 1(b), makes use of a single Cartesian IQ modulator, which is used to simultaneously generate the signal and optical carrier. This has a lower complexity, though has the drawback of limiting the voltage swing that can be used for the signal modulation drive waveform, particularly in the case of signals with large carrier-to-signal power ratios (CSPR). The third approach (Fig. 1(c)) makes use of a single dual-drive Mach Zehnder modular (DD-MZM). This offers the lowest complexity, though has the drawback that the optical signal bandwidth is limited to approximately half that with the IQ modulator, for a given electrical bandwidth. Despite this limitation, it is a promising approach for compact, low-cost integrated transmitters.

Surprisingly few experiments on multi-gigabit/s SSB signal generation using DD-MZMs have been reported. In [2], a $10 \mathrm{~Gb} / \mathrm{s}$ SSB-NRZ signal was transmitted over $320 \mathrm{~km}$ of standard SMF and electronic dispersion compensation was performed at the receiver. This experiment used analog electronics for the Hilbert filtering in the transmitter and the EDC. With the subsequent advances in D/A and A/D converter and DSP technologies, it was proposed to implement this scheme using digital signal processing [3]. In [4], a DD-MZM was used to generate and transmit 
$25 \mathrm{~Gb} / \mathrm{s}$ SSB-Nyquist-pulse-shaped 16-QAM signals over $500 \mathrm{~km}$ of uncompensated standard SMF. In [5], throughputs of 110 and $105 \mathrm{~Gb} / \mathrm{s}$ were achieved over 40 and $80 \mathrm{~km}$, respectively, using SSB discrete multitone (DMT) generated with a single DD-MZM, and a throughput of $180 \mathrm{~Gb} / \mathrm{s}$ over $13 \mathrm{~km}$ was reported in [6], using SSBduobinary-PAM-4. A summary of these experimental results is plotted in Fig. 2.

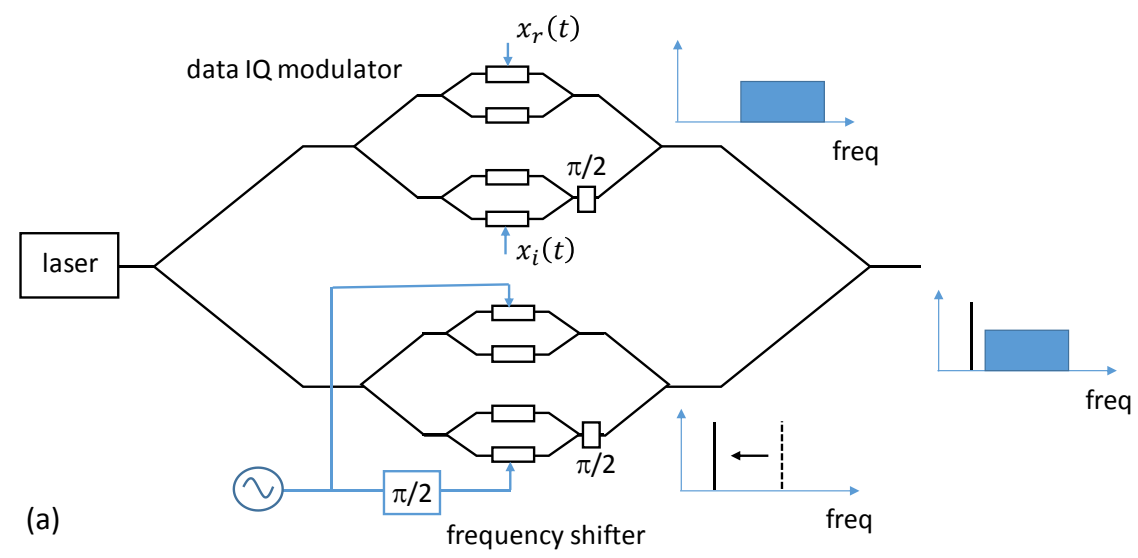

(b)

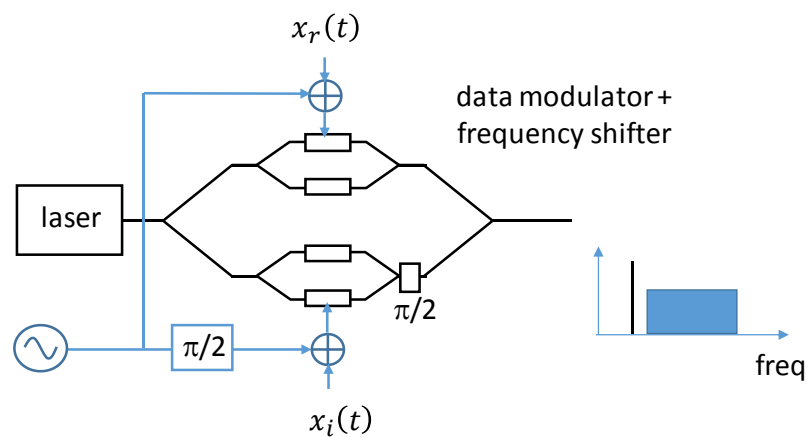

(c)

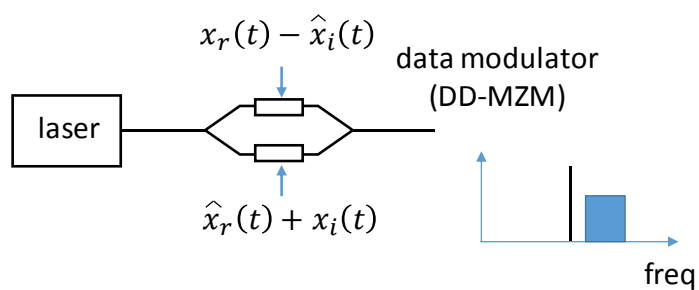

$\hat{x}$ - Hilbert transform of $x$

Fig. 1 SSB transmitter designs, using: (a) two IQ modulators, for data modulation and optical carrier generation, (b) a single IQ modulator for signal and carrier generation (with carrier frequency shifted), and (c) a dual-drive Mach-Zehnder modulator for signal and carrier generation (with optical carrier not frequency-shifted). $x_{r}$ and $x_{i}$-real and imaginary parts of the signal, respectively.

\section{Receiver linearization DSP}

Along with the signal generation, the linearization of the receiver also presents a challenge. Unless a large carrier-to-signal power ratio is used (which increases the required launch power, resulting in increased distortion due to fiber nonlinearity, and also increases signal-ASE beating noise), unwanted signal-signal beating terms need to be 
removed. A number of DSP-based approaches have been proposed, the most effective of which is the KramersKronig (KK) algorithm [1,7,8].

To date, the use of a DD-MZM-based transmitter for SSB signal generation, combined with receiver linearization DSP, such as the KK algorithm, has not been demonstrated, but is one approach which can potentially achieve low complexity, high performance self-coherent transmission systems, with high spectral efficiency and dispersion tolerance. It may be possible to exceed the performance previously achieved, plotted in Fig. 2 . Such lowcomplexity systems would be attractive for future metro and access networks and inter-data center links.

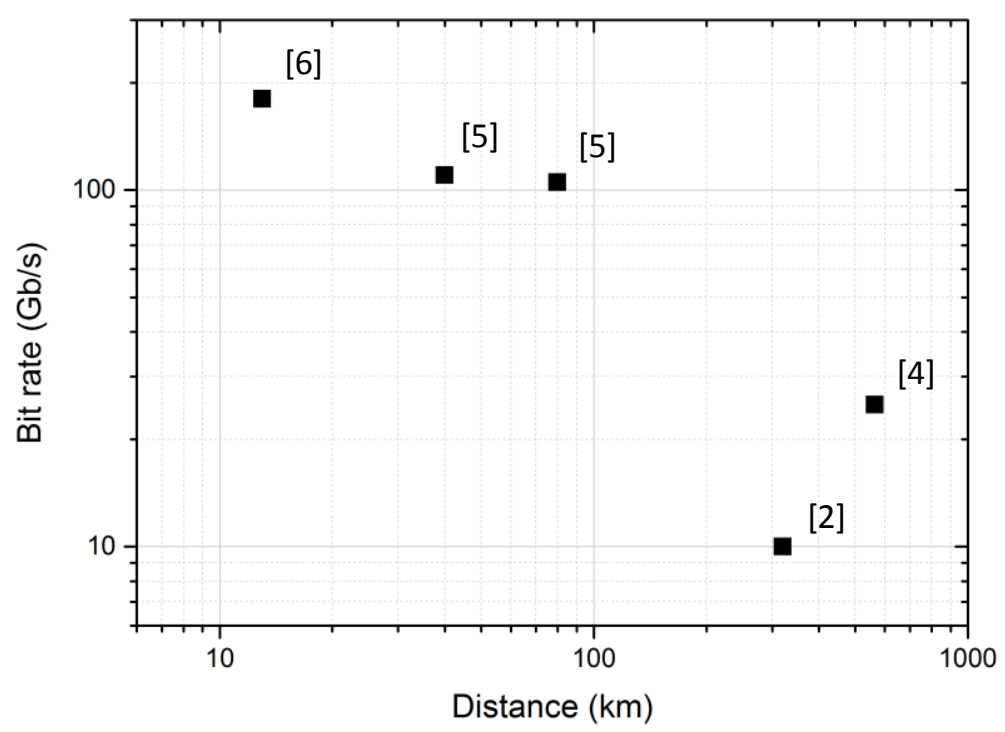

Fig. 2 Experimental results (throughput per wavelength versus transmission distance) reported for dual-drive Mach-Zehnder modulator-based single-sideband self-coherent systems.

This work was supported by the UK EPSRC UNLOC and TRANSNET Programme Grants.

\section{References}

[1] X. Chen, C. Antonelli, S. Chandrasekhar, G. Raybon, J. Sinsky, A. Mecozzi, M. Shtaif, and P. Winzer. 218-Gb/s single-wavelength, singlepolarization, single-photodiode transmission over $125-\mathrm{km}$ of standard singlemode fiber using Kramers-Kronig detection. In Optical Fiber Communication Conference (pp. Th5B-6), 2017.

[2] M. Sieben, J. Conradi, and D.E. Dodds. Optical single sideband transmission at $10 \mathrm{~Gb} / \mathrm{s}$ using only electrical dispersion compensation. Journal of Lightwave Technology, 17(10), p.1742, 1999.

[3] P.M. Watts, V. Mikhailov, M. Glick, P. Bayvel, and R.I. Killey. Single sideband optical signal generation and chromatic dispersion compensation using digital filters. Electronics Letters, 40(15), pp.958-960, 2004.

[4] M.S. Erkılınç, M.P. Thakur, S. Pachnicke, H. Griesser, J. Mitchell, B.C. Thomsen, P. Bayvel, and R.I. Killey. Spectrally efficient WDM Nyquist pulse-shaped subcarrier modulation using a dual-drive Mach-Zehnder Modulator and direct detection. Journal of Lightwave Technology, 34(4), pp.1158-1165, 2016.

[5] L. Zhang, T. Zuo, Y. Mao, Q. Zhang, E. Zhou, G.N. Liu, and X. Xu. Beyond 100-Gb/s transmission over 80-km SMF using direct-detection SSB-DMT at C-band. Journal of Lightwave Technology, 34(2), pp.723-729, 2016.

[6] Q. Zhang, N. Stojanovic, T. Zuo, L. Zhang, C. Prodaniuc, F. Karinou, C. Xie, and E. Zhou. Single-lane 180 Gb/s SSB-duobinary-PAM-4 signal transmission over 13 km SSMF. In Optical Fiber Communication Conference (pp. Tu2D-2), 2017.

[7] A. Mecozzi, C. Antonelli, and M. Shtaif. Kramers-Kronig coherent receiver. Optica, 3(11), pp.1220-1227, 2016.

[8] Z. Li, M.S. Erkılınç, K. Shi, E. Sillekens, L. Galdino, B.C. Thomsen, P. Bayvel, and R.I. Killey. SSBI mitigation and the Kramers-Kronig scheme in single-sideband direct-detection transmission with receiver-based electronic dispersion compensation. Journal of Lightwave Technology, 35(10), pp.1887-1893, 2017. 